\title{
Industrial frequency single-phase AC traction power supply system for urban rail transit and its key technologies
}

\author{
Qunzhan $\mathbf{L i}^{1}$
}

Received: 5 December 2015/Revised: 29 February 2016/Accepted: 3 March 2016/Published online: 6 April 2016

(C) The Author(s) 2016. This article is published with open access at Springerlink.com

\begin{abstract}
To avoid stray current and maintain the benefit of no phase-split in the DC traction power supply system, an $\mathrm{AC}$ traction power supply system was proposed for the urban public transport such as metro and light rail transit. The proposed system consists of a main substation (MSS) and cable traction network (CTN). The MSS includes a single-phase main traction transformer and a negative-sequence compensation device, while the CTN includes double-core cables, traction transformers, overhead catenary system, rails, etc. Several key techniques for the proposed system were put forward and discussed, which can be summarized as (1) the power supply principle, equivalent circuit and transmission ability of the CTN, the cable-catenary matching technique, and the selection of catenary voltage level; (2) the segmentation technology and status identification method for traction power supply network, distributed and centralized protection schemes, etc.; (3) a power supply scheme for single-line MSS and a power supply scheme of MSS shared by two or more lines. The proposed industrial frequency single-phase AC traction power supply system shows an excellent technical performance, good economy, and high reliability, hence provides a new alternative for metro and urban rail transit power supply systems.
\end{abstract}

Keywords Urban rail transit $\cdot 50 \mathrm{~Hz}$ industrial frequency single-phase AC traction power supply $\cdot$ Cable traction

The Chinese version of this paper was published in Journal of Southwest Jiaotong University (2015) 50(2).

Qunzhan Li

lqz3431@263.net

1 School of Electrical Engineering, Southwest Jiaotong University, Chengdu 610031, China network $\cdot$ Power supply state monitoring and control $\cdot$ Main substation $\cdot$ Stray current

\section{Introduction}

From worldwide application point of view, DC system, low-frequency single-phase AC system, and industrial frequency single-phase AC system are three main power supply systems for trunk railways, metros, and light rails. Each of them has its own characteristics and some comparative advantages changing with the development of electrotechnics [1]. Today, except the low-frequency single-phase AC system still being used in trunk railways in Germany and some of its neighborhood countries, the DC system and the industrial frequency single-phase AC system have been mainly used around the world. The DC system is mainly used for metros and light railways, while the industrial frequency single-phase AC system is mostly for trunk railways [2-4], both with an increasing automation level [4-6].

Metros and light railways in urban transit systems have prominent features of large traffic density and frequent starts and stops. Their DC traction power supply systems have the advantage of no phase-split along the overhead catenary system (OCS), so that trains can run smoothly. On the other hand, DC traction systems also have the disadvantage of the long-term adverse effects caused by the stray current (earth fan stream) [3,6]. Anti-stray current measures, however, are usually complex with high costs and limited effects because the stray current is hard to control. After 1960s, except that parts of the trunk railway lines still retain the DC power system in Europe and the former Soviet Union, almost all of newly built trunk railways, especially high-speed railways or heavy-haul 
railways, have adopted the industrial frequency singlephase AC system, because of its advantages of strong supply capacity, low costs, being able to meet the large transport capacity requirements, non-existence of stray current, and directly efficient utilization of regenerative braking power. However, its disadvantages, switched into the public power grid in order to reduce impacts of negative-sequence component, create the phase-split in traction power supply side, which leads to power supply breakpoints for running trains. Therefore, techniques for auto-passing phase-splits have to be developed due to the increasing speed of trains. Another drawback is that the harmonic current of traction load could produce electromagnetic interference in the vicinity of communication lines, even though with the use of optical cables it is not a problem for the protection to interfere.

Traction motors, as the power source of trains [7], can be categorized both as DC and AC types. Compared with a $\mathrm{DC}$ traction motor, an $\mathrm{AC}$ traction motor has properties of high-power density, low costs, simple and reliable structure, good anti-idling performance, etc. In addition, the absence of mechanical parts in DC traction motors, such as the commutator, makes AC traction motors more suitable for operation of high-power, high-speed trains. Therefore, the AC traction motor is replacing the DC traction motor and has become the main power source for trunk railways and urban rail transit.

The train, powered by AC traction motors and composed of an electric locomotive or electric multiple units (EMUs), is called an AC-DC-AC train, which is developed mainly for trunk railways and high-speed railways. It is easy for AC-DC-AC trains to store the brake energy back to the grid, it is not only simple, convenient, safe but also reliable to operate. In contrast, for a DC traction motor, to achieve these goals, very special and expensive equipment and complex controlling devices have to be extra utilized, and some of these goals are even unachievable ultimately.

Considering the characteristics of the traction power supply system for trunk railways, metros, and light rails in urban transit system, and comparing the merits and demerits between the DC system and the industrial frequency single-phase AC system, it is naturally necessary to provide a new industrial frequency single-phase AC traction power supply system without phase-split or stray current in urban transit system.

Compared to trunk railways, the power supply lines of metros and light rails are shorter, usually less than $50 \mathrm{~km}$, and does not have line-crossing or line-joining. To achieve an AC traction power supply system for metros and light rails without phase-split, a direct approach would be used to co-phase power supply technique that was proposed for trunk railways [8-10] and the recently developed traction power supply system of new generation [11]. However, the power supply system for metros and light rails is significantly different from that for trunk railways, because it has shorter power supply line, lower voltage grade, and denser traction substations. In order to reduce the impact of negative-sequence current, negative-sequence compensation devices have to be installed at every traction substation. Additionally, bilateral supply mode needs to be carried out at section posts (between two adjacent substations) by connecting different power supply lines at the high-voltage side, which is known as loop closing [12, 13]. Feasibility study must be carried out after obtaining consent from power sectors. To avoid complications and difficulties mentioned above, a new industrial frequency-based singlephase AC traction power supply system using $35 \mathrm{kV}$ cables is proposed in this paper for urban rail transit, due to the fact that $35 \mathrm{kV}$ cables have widely been used in metro systems.

This study is focused on the proposed AC traction power supply system and its key technologies, which include cable traction network, segmented power supply, status identification and protection, and main substation power supply scheme. In addition, a brief discussion on system's economy and reliability, etc. is also given.

\section{Cable traction network}

The proposed industrial frequency single-phase AC traction power supply system for the urban rail transit consists of a main substation (MSS) and cable traction network (CTN). The latter, as shown in Fig. 1, comprises the cables, traction transformers (TT), overhead catenary system (OCS), rails (R), etc. The type of the cables can be a twocore cable (TCC), or a coaxial cable (CC). TCC is made of a supply core (SC), a return core (RC), and a protective layer (PL). The TT has single-phase connection. The TCC and the OCS are erected in parallel. The primary winding of the TT is connected in parallel between the SC and RC of TCC, while its secondary winding is connected in parallel between the OCS and the R. TTs are distributed in proper distances along TCC and OCS. The SC of TCC is connected with the OCS in parallel by adjacent TTs, so are the RC of TCC and the R. Electric trains (ET), either electric locomotives or EMUs, are powered between the OCS and the R. In Fig. 1, BB1 and BB2 represent main traction busbars; $\mathrm{W}$ and $\mathrm{K}$ represent circuit breakers; $\mathrm{S}$ represents OCS slicer; P represents section post; and ET represents an electric train, which is an electric locomotive or an EMU. MSS and its power supply scheme will be discussed in Sect. 3.

The primary side of MSS should be connected to threephase lines in a utility grid of 110 or $220 \mathrm{kV}$, while the secondary side should use $35 \mathrm{kV}$ TCC for traction power 


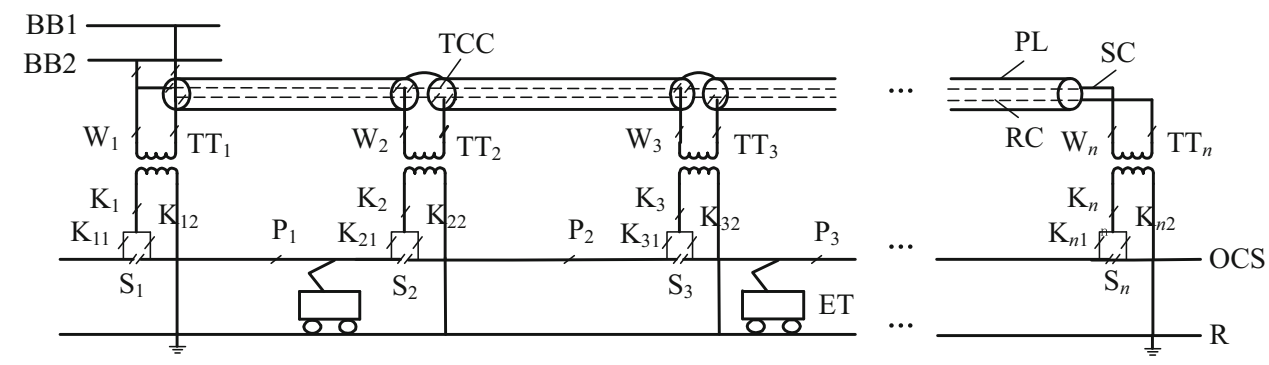

Fig. 1 Traction power supply network with TCCs

supply and use $35 \mathrm{kV}$ three-phase cables for three-phase power and single-phase lighting supply. The single-phase traction power supply and the three-phase power and lighting supply should be kept independent to avoid the direct impact of negative sequence generated in traction power supply on the power and lighting system, also avoid the mutual interference between the two systems during operation and maintenance.

The transmission capacity refers to the maximum transmission power delivered through cables, overhead lines, catenaries, or other types of transmission lines [14]. The natural power is one of the most important characteristic parameters for measuring the transmission capacity. It is defined as the transmitted power when the load impedance at the transmission line terminal is equal to the wave impedance. Given the distributed resistance $R_{0}$, the distributed inductance $L_{0}$, the distributed capacitance $C_{0}$ between lines, the distributed conductance $G_{0}$, as to per unit length of cables, overhead lines or other transmission lines, the wave impedance $z_{\mathrm{c}}$ (modulus value) can be calculated by [15]

$z_{\mathrm{c}}=\sqrt{\left(R_{0}+\mathrm{j} \omega L_{0}\right) /\left(G_{0}+\mathrm{j} \omega C_{0}\right)}$,

where $\mathrm{j}$ is the imaginary unit $\left(\mathrm{j}^{2}=-1\right)$, and $\omega$ is angular frequency.

Under the industrial frequency, there are $R_{0} \ll \omega L_{0}$ and $G_{0} \ll \omega C_{0}$, then the wave impedance of a lossless transmission line is obtained as

$z_{\mathrm{c}} \approx \sqrt{L_{0} / C_{0}}$

It can also be said that the wave impedance of a lossless transmission line is a pure resistance. In this case, the electric field energy released (or absorbed) from the distributed capacity $C_{0}$ is equal to the magnetic field energy absorbed (or released) from the distributed reactance $L_{0}$. That is to say, the reactive power of the distributed capacity and the distributed reactance are just balanced. Thus, the transmitted power is completely equal to the load active power. If the rated voltage is $U_{\mathrm{N}}$ and the wave impedance (modulus value) is $z_{\mathrm{c}}$, the natural power of a transmission line is
$S_{\mathrm{c}}=U_{\mathrm{N}}^{2} / z_{\mathrm{c}}$.

The natural power is often used for analyzing the transmission condition, voltage level, etc. of a transmission line. When natural power is transmitted, the voltages at the two terminals of the line will be the same, and so are the power factors. When the transmitted power is lower than the natural power, because of the decreased transmission line current, the reactive power of the distributed reactance which is proportional to the square of the line current, will be less than the reactive power of the distributed capacity. This leads to a higher voltage at the line terminal. Similarly, when the transmitted power is larger than the natural power, due to the increased transmission line current, the reactive power of the distributed reactance will be larger than that of the distributed capacity and the voltage at the line terminal will be lower. Theoretically, it is ideal to transmit the natural power. This is hard to be achieved in practice, particularly when traction loads fluctuate sharply: the voltage increases at no load and drops at heavy load. In order to maintain a good transmission capacity, sometimes the inductive reactive power compensation is needed, or sometimes the capacitive reactive power compensation is required, even both are demanded at the same time.

By Eq. (1), the wave impedance decreases as the distributed capacitance increases, and increases as the distributed reactance increases. Usually the wave impedance of overhead lines is between 270 and $380 \Omega$ [14]. The mutual inductance approaches to the self-reactance when the cable cores are arranged closely. The equivalent distributed reactance is greatly reduced when the values of currents in two cores are the same with opposite directions. At the same time, when the distance between the cable cores is closer and the insulation material between the cores has a relative dielectric constant up to 4 or 5 , the distributed capacitance is greatly increased. So the cable's wave impedance would be much less than overhead line's impedance. The cable's wave impedance would be approximately $1 / 7$ of overhead line's impedance [15]. From Eq. (2), it can be seen that the transmission capability of a cable is approximately 7 times those of overhead lines 
or catenaries at the same voltage level, since the natural power is inversely proportional to the wave impedance. Empirical data [14] show that the output power at one side of a single loop (head-to-end) of $35 \mathrm{kV}$ overhead lines can reach up to $10 \mathrm{MW}$ with a transmission distance of approximately $20 \mathrm{~km}$, while the output power of cables under the same condition can reach up to $70 \mathrm{MW}$ (for double-loop circuit lines to $140 \mathrm{MW}$ ). Thus it can be derived that the cable's one-side transmission distance is up to $100 \mathrm{~km}$ or longer. It is able to fully meet the needs of any metro or light rail line.

It should be noted that power transmission may have different needs, and the transmission capacity is affected by many factors. In addition to the natural power, other factors such as economic factor, thermal stability limit, voltage drop limit, system stability, etc. [14], should also be taken into account so as to find out an optimal solution.

In current metro and light rail, 1,500 V DC voltage is adopted for the OCS with an exception of $750 \mathrm{~V}$ in particular cases. If the OCS uses an industrial frequency AC system, its voltage grade will be determined by the following factors: (1) AC peak voltage, (2) tunnel clearance and OCS insulation requirement, (3) transformerless ACDC-AC ET, and so on. Selecting a voltage grade as high as possible will improve the OCS's power supply capacity and reduce the power loss. In general, a feasibility study of the OCS voltage (RMS value) between 2 and $3.5 \mathrm{kV}$ should be carried out.

Figure 2 shows a chain network, which is the equivalent circuit of CTN illustrated in Fig. 1. The OCS-R loop can be imputed to the cable loop side, or the cable loop can be imputed to the OCS-R loop side.

In Fig. 2, the thick solid line $Z_{1 i}$ represents the distribution parameter of the $i$-th segment of the cable loop; the thick solid line $Z_{2 i}^{\prime}$ represents the distribution parameter of the OCS-R loop, which is imputed to the cable loop side; the traction transformer is considered as a lumped parameter element and its leakage reactance imputed to the cable loop side is represented by $Z_{\mathrm{T} i}$. Taking into account the compactness of TCC and the isometry between TCC loop and OCS-R loop, the coupling between TCC loop and OCS-R loop can be ignored generally in the analysis and calculation. To further simplify the calculation, the lumped parameters can be used to establish the equivalent circuit of OCS-R loop.

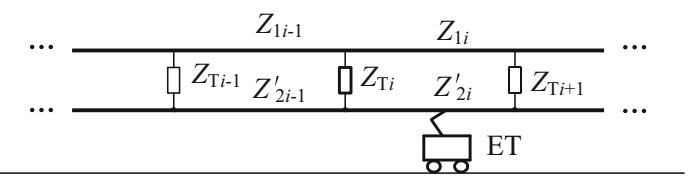

Fig. 2 Equivalent circuit of traction network
The current of TCC loop or OCS-R loop is inversely proportional to the loop impedance. From the above analysis about the wave impedance, it can be inferred that the TCC loop impedance will be much smaller than the OCS-R loop impedance and the current in TCC will be larger. Moreover, if OCS-R loop is imputed to TCC loop side, the imputed impedance modulus value of OCS-R loop will be the square of its turn ratio. For example, when the TT primary voltage is $35 \mathrm{kV}$, its turn ratio would be no less than 10 if the chosen OCS voltage does not exceed $3.5 \mathrm{kV}$, and the impedance modulus value of TCC loop will be less than $1 \%$ of the impedance modulus value of OCS-R loop imputed to TCC loop side. The OCS-R loop between two nearest TTs can be called short loop (the $i$-th segment in Fig. 2). And the parallel connection of the TCC loop and the OCS-R loop from the short loop to the main traction busbar can be called long loop (from the 1st to the $(i-1)$ th segments in Fig. 2). If so, the short loop will take the train load while the OCS on the long loop will hardly bear any burden for power supply (less than $1 \%$ ). The TCC on the long loop will bear almost all power supply burden (larger than $99 \%$ ). That is, the OCS on the short loop is mainly responsible for the power supply of the train in the current segment, and the TCC on the long loop is responsible for the power supply of the entire traction network.

In [16], analyses and calculations of a coaxial-cable traction power supply network are given for reference.

Metro or light rail's upward and downward traction network works in parallel. Besides, auxiliary cables should be set up to work with the main cable at the same time, which means that they are reserved for each other. This can help improving the power supply capacity and reducing the power loss and voltage loss of the traction network.

\section{Traction power supply segmentation, status identification, and protection}

As mentioned in Sect. 1, the CTN is characterized by strong transmission capacity and long distance transmission ability. However, its construction is complex, and particularly more complex when the upward and downward network together with auxiliary cables are working in parallel. Based on the analyses and experimental studies of the control and monitoring for all-parallel AT (auto-transformer) power supply mode of the high-speed railway in China [11, 17], the segmented power supply has become an effective approach for timely removal of faults, which limit faults to a minimum scope so that failure effects can be reduced to the minimum. The segmented power supply avoids the scenario in which one failure could lead to 


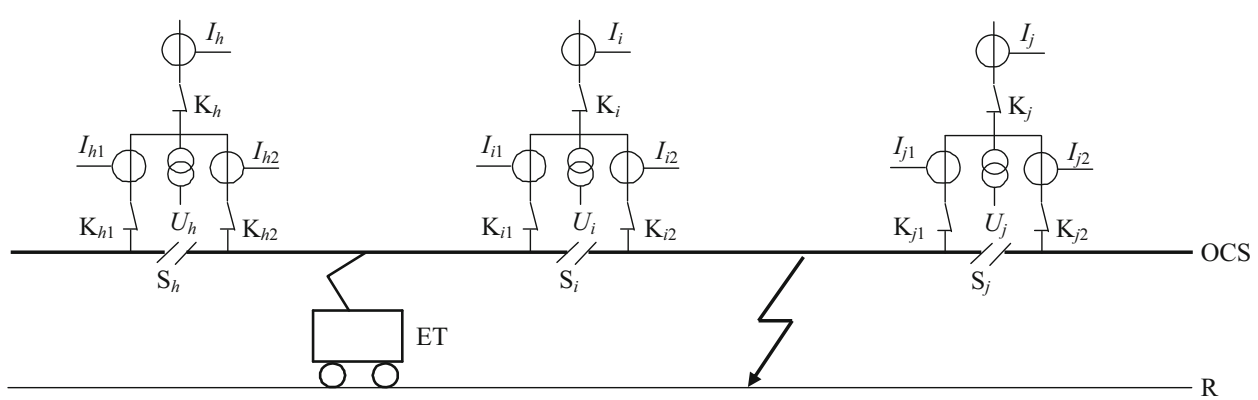

Fig. 3 Diagram of SS feeder and OCS segments

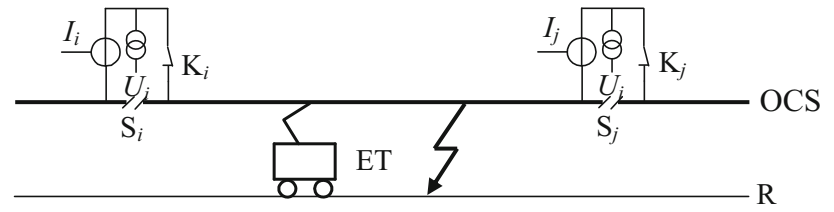

Fig. 4 OCS segments diagram

system failure and thus jeopardize the system's reliability and availability.

Cables and OCSs can be segmented separately or segmented at a common location, for convenient purpose, lumped segment is better to be at the location of a TT where the traction substation (SS) is built. The SS $i$ includes $35 \mathrm{kV}$ entrance cables, a main tie line, a TT, traction busbars, feeder lines, a feeder breaker $\mathrm{K}_{i}$, a subfeeder, and its circuit breaker, etc. At the exit of SS $i$, there should be a nearby OCS slicer $S_{i}$ which is in series with OCSs, so as to allow a train with uninterrupted supply. The SS $i$ should also include the sub-feeder breakers $K_{i 1}$ and $\mathrm{K}_{i 2}$ connected in parallel with $\mathrm{S}_{i}$, the current transformer (measured currents denoted by $I_{i 1}$ and $I_{i 2}$ ), and the voltage transformer connected to the OCS slicer $S_{i}$ (measured voltage denoted by $U_{i}$ ). The segmented power supply at SS feeder lines and OCS segments is shown as Fig. 3.

The distance between two adjacent SSs, in reference to existing metros or light rail lines, is typically $2-4 \mathrm{~km}$. If the OCS's length between SSs is considered too long, it can be more segmented, as shown in Fig. 4.

The power supply of a traction network has two states: normal state and faulty state. The former includes no-load state and normal load state while the latter includes the short circuit and the open circuit (the broken line). These states can be identified by measuring the voltage from voltage transformer or the current from current transformer. Based on the fact that the cable's failure rate is much lower than the OCS's failure rate in a traction network, even the power supply through the OCS is more complex than that through the cable, the main focus in this paper will be given to the OCS segmented power supply, status identification, and protection methods.
Assumptions: When the local OCS voltage (a modulus value, the same applies hereinafter) $U_{i} \geq U_{\mathrm{s}}$ (a specified value), it is in the normal power supply state on the left and right segments of the OCS. If $U_{i}<U_{\mathrm{s}}$, then the train on the left and right segments of the OCS is not in a working state; that is, the train load is zero and thus the OCS segment may be in a failure state.

Acquire the OCS voltage $U_{i}$ of the SS $i$ measured by the voltage transformer, the left-side OCS current $I_{i 1}$ and the right-side OCS current $I_{i 2}$ of the SS $i$ measured by the current transformer, where $i=1,2, \ldots, N$. For a OCS segment between SS $i$ and $\operatorname{SS} j(j=i+1)$ as shown in Fig. 3, the operation state of a train can be identified by calculating the arithmetic difference of currents between the two ends of the OCS segment if the following assumptions are satisfied [11, 17]: when the OCS voltages $U_{i}, U_{j} \geq U_{\mathrm{s}}$, the current $I_{i 2}$ that flows into the segment and the current $\dot{I}_{j 1}$ that flows out of the segment are all positive, their power factors are the same, and the OCS-to-ground distributed capacitive current and the measurement error are ignored, who are relatively small.

If $I_{i 2}-I_{j 1}=0$, there is no electric train in the segment; when $I_{i 2}=I_{j 1}=0$, the segment is on a no-load state; when $I_{i 2}=I_{j 1}>0$, the segment acts as a transmission line to pass transmission power.

If $I_{i 2}-I_{j 1}>0$, there is a moving train being in traction in the segment.

If $I_{i 2}-I_{j 1}<0$, there is a moving train being in regenerative breaking in the segment.

When the OCS voltage $U_{i}<U_{\mathrm{s}}$, according to the above assumptions, there would be no load within the adjacent OCS segments. So there is only short-circuit current. For the OCS segment between $\mathrm{SS} i$ and $\mathrm{SS} j(j=i+1)$ as shown in Fig. 3, it is still assumed that the current $I_{i 2}$ flowing into the segment is positive, also the current $I_{j 1}$ flowing out of the segment is positive, and their traction impedance angles are equal. Hence the differential current is

$I_{\mathrm{D} i 2}=\left|I_{i 2}-I_{j 1}\right|$.

Clearly, if there is no short circuit, then $I_{i 2}=I_{j 1}$, and 
$I_{\mathrm{D} i 2}=\left|I_{i 2}-I_{j 1}\right|=0$. If a short circuit occurs, then $I_{j 1}$ is reversed and $I_{\mathrm{D} i 2}=\left|I_{i 2}+I_{j 1}\right|>0$. This leads to a lowvoltage starting differential current protection for OCS. Specifically, acquire the OCS voltage $U_{i}$, the left-side OCS current $I_{i 1}$, the right-side OCS current $I_{i 2}$, the right-side OCS current $I_{h 2}$ on the left adjacent substation $h$ $(h=i-1)$ and the left-side OCS current $I_{j 1}$ on the right adjacent substation $j(j=i+1)$. The OCS differential current at the right-side of substation $i$ is given by Eq. (3) and the one at the left-side of the substation $i$ is

$I_{\text {Di1 }}=\left|I_{i 1}-I_{h 2}\right|$.

When $U_{i}<U_{s}$ at substation $i$, if the left-side OCS differential current $I_{\mathrm{D} i 1}>0$, it can be concluded that there is a short-circuit fault on the OCS between SS $i$ and the left adjacent SS $h$. Then, open the breaker $\mathrm{K}_{i 1}$ at SS $i$ and the breaker $\mathrm{K}_{h 2}$ at the SS $h$, otherwise both breakers $\mathrm{K}_{i 1}$ and $\mathrm{K}_{h 2}$ are still in closed state. Similarly, if the right-side OCS differential current $I_{\mathrm{D} i 2}>0$ at SS $i$, it can be concluded that there is a short-circuit OCS fault between SS $i$ and the right adjacent $S S j$, then open breakers $\mathrm{K}_{i 2}$ and $\mathrm{K}_{j 1}$ for the same reason.

Further analysis reveals that if one of the currents in $\left\{I_{i 1}, I_{h 2}\right\}$, or $\left\{I_{i 2}, I_{j 1}\right\}$ is zero, then at the side where the current is zero, an open-circuit fault has occurred, while at another side where the current is not zero, a short-circuit fault has occurred.

At the same time, the short-circuit impedance can be calculated from the OCS's voltage and current in a faulty state, subsequently the fault location can be identified.

The setup of low-voltage starting differential current protection of OCS at SS $i$ is to meet the quick-action requirement. It is only dependent on the local SS and two adjacent SSs. The train operational state identification is required by the MSS or the dispatching center, and should cover every SS in the entire traction network [5]. The MSS or the dispatching center can set up backup protection based on acquired OCS voltages and currents from each of SSs. Due to these differences, the low-voltage start and differential current protection at each SS is called distributed protection, while the backup protection at MSS or the dispatching center is called centralized protection.

In order to improve the real-time performance of the centralized protection, the sign value method can be utilized [11, 17], which is based on the fault power flow direction. The fault power flow is defined as the current phasor under the unit voltage as the reference phasor. Set the sign value to 1 if the direction of the fault power flows into the segment, to -1 if it flows out of the segment, and to 0 for no-load fault power flow. Calculate the sign value of fault power flow into the current segment and its absolute value $p$, faults can be identified as follows:
If $p=0$, there is no short-circuit fault at the current segment.

If $p=1$, there is a short-circuit fault at one current segment end if the absolute value of the sign value is 1 ; there is an open-circuit fault at the other end if the absolute value of the sign value is 0 .

If $p=2$, there are short-circuit faults at both ends of the current segment.

Obviously, this result is consistent with the result obtained from the low-voltage starting differential current protection approach mentioned previously. In addition, the method of fault current sign value is easier to further determine the OCS's composite faults.

The segmented power supply and protection for OCSs can greatly improve the reliability of the system [14]. This technique is also applicable to the protection of the cable segmented supply in a similar way.

It is evident that the use of traction network segmentation technique allows more flexibility for the traction network's operation and maintenance.

\section{Main substation power supply scheme}

The scheme of an MSS power supply is dependent on the number and the relative position of metro or light rail line that requires the power supply.

\subsection{One line case}

If only one metro or light rail line is needed by the power supply, the main substation (MSS) should be located as close to the middle of the line as possible, as shown in Fig. 5, where MSS provides power for both sides of the line. If the condition doesn't permit, the MSS can be placed at one line end and thus uses unilateral power supply as shown in Fig. 1. In both arrangements, the MSS supplies the power by combining a single-phase main transformer (MTT) with a negative-sequence compensation device (NCD), to realize the centralized negative-sequence management as shown in Fig. 6. This scheme also includes a transformer for three-phase power and single-phase lighting supply (not shown in the diagram, the same applies hereinafter). The NCD consists of a high-voltage matching transformer (HMT), an AC-DC-AC converter (ADA), a traction matching transformer (TMT), and so on. The length of the bilateral power supply doubles that of the unilateral power supply. If the supply's length is the same, the bilateral supply can save cable material and reduce power loss and voltage loss.

It should be noted that the negative-sequence compensation mentioned here is referred to as the symmetrical compensation in a broad sense. As stated in [10], the 


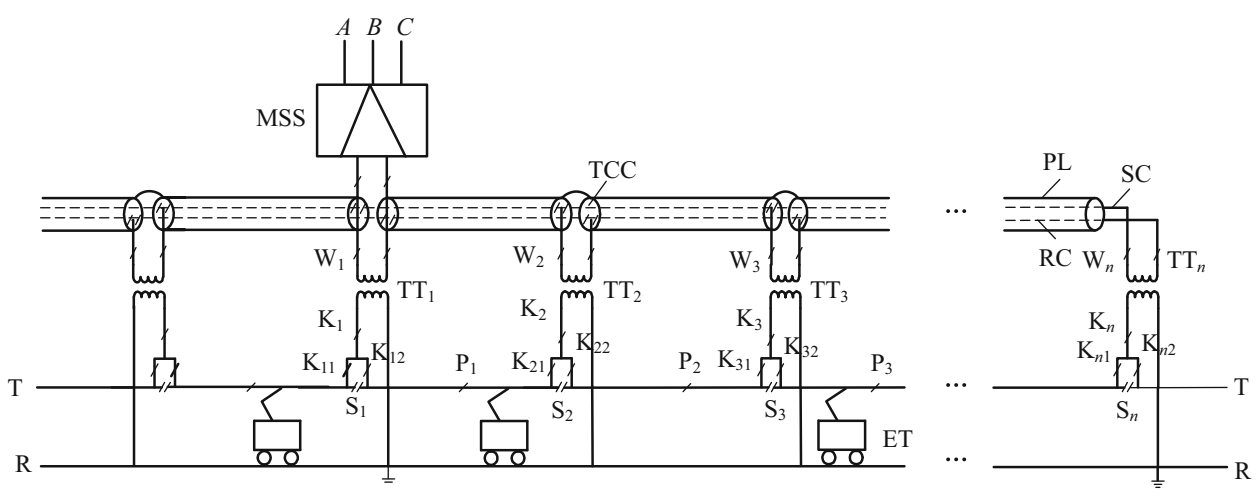

Fig. 5 Traction power supply system diagram

symmetrical compensation includes negative-sequence compensation and reactive power compensation based on three-phase to one-phase symmetrical transformation. Since the power factor of an AC-DC-AC train is close to 1 , the reactive power compensation has been achieved, and just the negative-sequence compensation has become the main target of compensation. Thus the symmetrical compensation can be termed as the negative-sequence compensation. In the same principle as applied in a co-phase compensation device [11], the NCD needs to transfer active power to compensate negative sequence. If necessary, functionalities of reactive power compensating and harmonic filtering are able to be realized at these device terminals.

If the short-circuit capacity of a utility grid connected with the MSS is denoted by $s_{\mathrm{sc}}$ (MVA), and the three-phase voltage unbalance value $(95 \%$ probability value or the maximum) specified by the national standard in China [18] (GB for short, the same applies hereinafter) is denoted by $u_{\varepsilon}(\%)$, then the allowable value of the negative-sequence power, that is $s_{\varepsilon}$, is given by

$s_{\varepsilon}=u_{\varepsilon} \times s_{\mathrm{sc}} / 100$.

Using the load process $s_{\mathrm{L}}(t)$ obtained from power supply calculation, we can work out the load power $s$ corresponding to the GB specified value (95\% probability value or the maximum). Then, the calculated MTT capacity $s_{\mathrm{T}}$ and the calculated NCD capacity $s_{\mathrm{C}}$ are obtained as follows [11]:

$\left[\begin{array}{l}s_{\mathrm{T}} \\ s_{\mathrm{C}}\end{array}\right]=\frac{1}{2}\left[\begin{array}{cc}1 & 1 \\ 1 & -1\end{array}\right]\left[\begin{array}{c}s \\ s_{\varepsilon}\end{array}\right]$.

Further determination of their installed capacities should base on the overload characteristics of MTT and NCD.

The MSS should be equipped with backup devices as demonstrated in Fig. 7. In addition to the devices shown in Fig. 6, a backup MTT (BMTT) and a backup HMT (BHMT) are added. To save space, the MTT and the HMT can be fabricated into one box, so can the BMTT and the

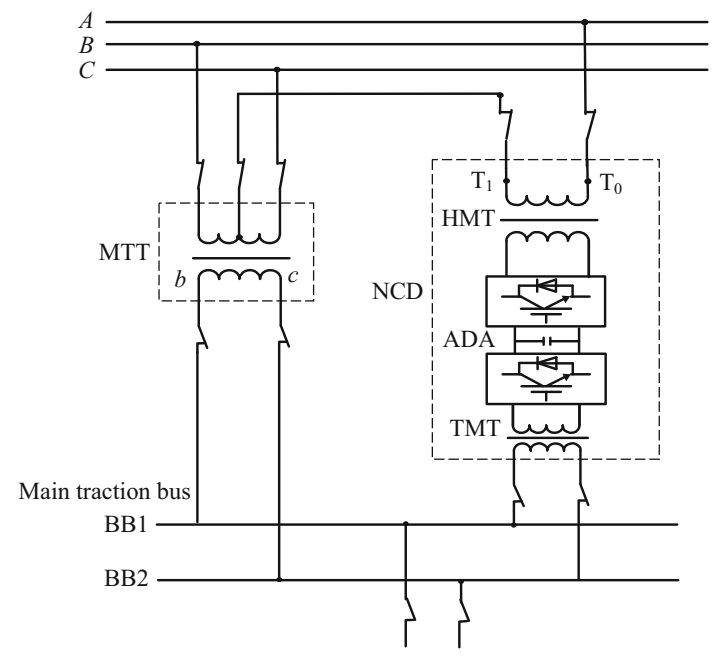

Fig. 6 MSS connection for one line

BHMT. The NCD adopts an $m / n$ system; that is to say, among $n$ units, $m$ units work while $n-m$ units act as backup units. Figure 7 shows a 3/4 system or a 2/4 system.

\subsection{Two line case}

If an MSS needs to supply power for two independent metro or light rail lines whose trains can't cross each other, the three-phase to two-phase balanced traction transformers [7] should be utilized as in a trunk railway line to reduce the impact of negative sequence. However, considering their scalability and dynamic negative-sequence compensation, it is better to build a combination scheme based on the system shown in Fig. 6. To reduce negative-sequence impact and lower down NCD costs, only two of the three phases are used for power supply, as shown in Fig. 8. In this figure, the primary side of the main traction transformer $\mathrm{MTT}_{A B}$ is connected to phase $A$ and phase $B$ of a utility grid, where $U_{A B}$ is the line voltage, and the secondary side feeder $\mathrm{F}_{a b}$ supplies power for one line. 


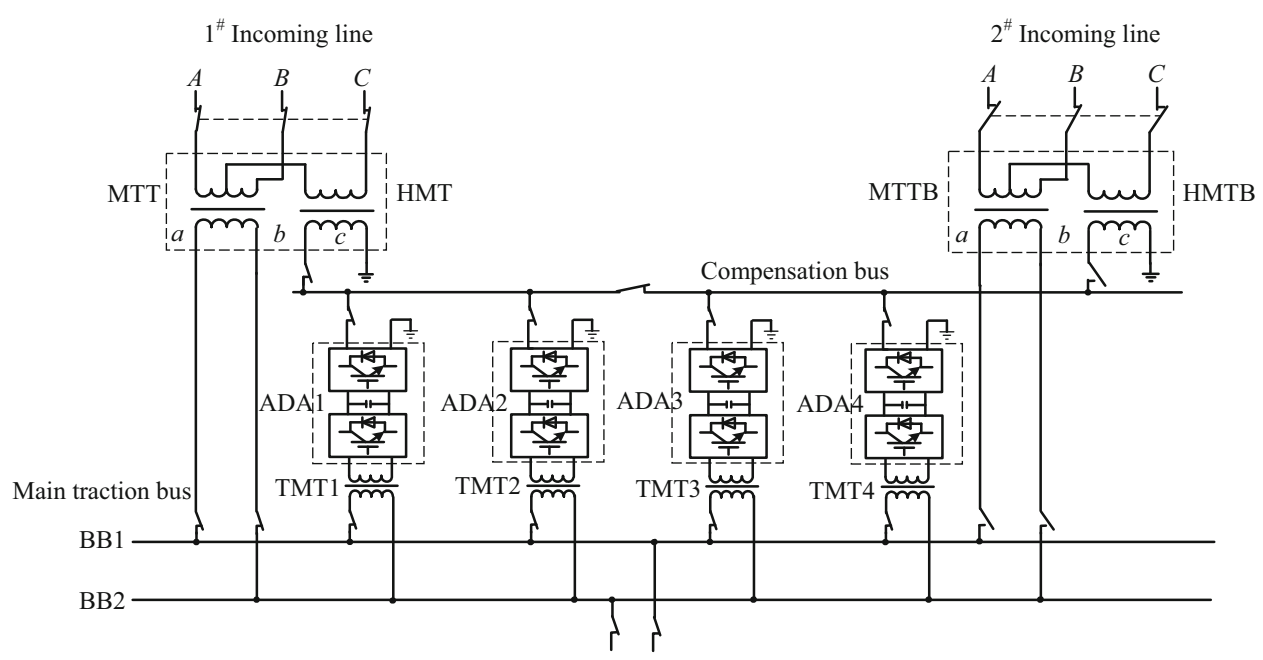

Fig. 7 MSS connection and standby equipments for one line

Similarly, the primary side of the main traction transformer MTT $_{B C}$ is connected to phase $B$ and phase $C$ of a utility grid, where $U_{B C}$ is the line voltage and the secondary side feeder $\mathrm{F}_{b c}$ supplies power for another line. The YNd11connected three-phase high-voltage matching transformer HMT is connected to two NCDs, where AC-DC-AC converters are $\mathrm{ADA}_{a}$ and $\mathrm{ADA}_{c}$, respectively, $\mathrm{ADA}_{a}$, $\mathrm{MTT}_{B C}$, and $\mathrm{F}_{b c}$ form one group, while $\mathrm{ADA}_{c}, \mathrm{MTT}_{A B}$, and $\mathrm{F}_{a b}$ form another group; $\Delta, \bullet$, and $*$ represent the dotted terminals.

In general, the traction power is larger than the regenerative power on one line; that is, regenerative power will be absorbed by the train in traction. For this reason, pure regenerative power will be ignored in calculating capacities of the MTT and the negative-sequence compensation. Denote the traction load process $s_{\mathrm{L} a b}(t)$ of $\mathrm{F}_{a b}$ and the traction load process $s_{\mathrm{L} b c}(t)$ of $\mathrm{F}_{b c}$ as $s_{a b}$ and $s_{b c}$ correspondingly to the GB-specified values $(95 \%$ probability value or the maximum). Assuming that their load power factors are the same, if the power of two lines is supplied with the same line voltage as shown in Fig. 6, the synthesis of negative sequence can be expressed as Eq. (6).

$s_{1}^{-}=s_{a b}+s_{b c}$.

If the power of the two lines is supplied with two different line voltages as shown in Fig. 8, the negativesequence modulus value based on 120 degree synthesis will be less than or equal to the larger one between $s_{a b}$ and $s_{b c}$, that is,

$s_{2}^{-} \leq \max \left\{s_{a b}, s_{b c}\right\}$.

Comparing Eq. (6) with Eq. (7), we can see that the negative-sequence power and the NCD capacity are lowered down greatly in Eq. (7). The maximum value is

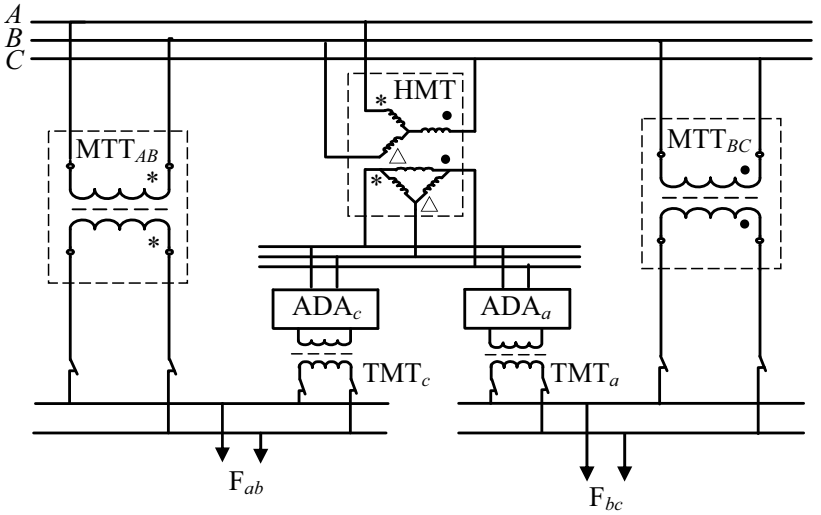

Fig. 8 MSS connection for two lines

achieved at $s_{a b}=s_{b c}$ so that the NCD capacity can be decreased by $50 \%$.

According to the GB [18], the MSS with two lines as shown in Fig. 8 is assessed through synthesis of negativesequence components. The compensation power portions are decomposed into the two NCDs according to proportions of load power $s_{a b}$ and $s_{b c}$, respectively. Each of the NCDs only works in accordance with its feeder's traction load. That is to say, the $\mathrm{ADA}_{a}$ is a current source controlled by $s_{\mathrm{L} b c}(t)$ of $\mathrm{F}_{b c}$. Similarly, the $\mathrm{ADA}_{c}$ is a current source controlled by $s_{\mathrm{L} a b}(t)$ of $\mathrm{F}_{a b}$. The calculated capacities of $\mathrm{MTT}_{B C}$ and $\mathrm{ADA}_{a}$ can be obtained by substituting $s=s_{b c}$ into Eq. (5). Likewise, the calculated capacities of $\mathrm{MTT}_{A B}$ and $\mathrm{ADA}_{c}$ can be obtained by substituting $s=s_{a b}$ into Eq. (5).

\subsection{Three lines case}

In order to minimize the impact of negative sequence and reduce the NCD capacity and costs, apparently three metro 


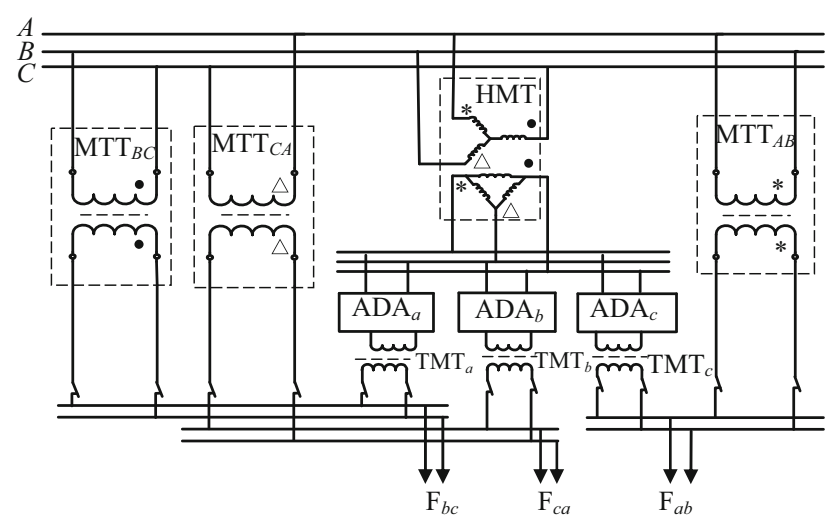

Fig. 9 MSS connection for three lines

or light rail lines should be powered with three different line voltages as shown in Fig. 9. Here, the primary side of $\mathrm{MTT}_{A B}$ is connected to phase $A$ and phase $B$ of a utility grid, and $\mathrm{F}_{\mathrm{ab}}$ in the secondary side supplies the power for the first line. The primary side of $\mathrm{MTT}_{\mathrm{BC}}$ is connected to phase $B$ and phase $C$, and $\mathrm{F}_{b c}$ in the secondary side supplies the power for the second line. The primary side of $\mathrm{MTT}_{C A}$ is connected to phase $C$ and phase $A$, and $\mathrm{F}_{c a}$ in the secondary side supplies power for the third line. The YNd11connected three-phase HMT is used to link three NCDs, where $\mathrm{AC}-\mathrm{DC}-\mathrm{AC}$ converters are $\mathrm{ADA}_{a}, \mathrm{ADA}_{b}$, and $\mathrm{ADA}_{c}$, respectively. Thereinto, $\mathrm{ADA}_{a}, \mathrm{MTT}_{B C}$, and $\mathrm{F}_{a b}$ form the first group. $\mathrm{ADA}_{b}, \mathrm{MTT}_{C A}$, and $\mathrm{F}_{C A}$ form the second group. $\mathrm{ADA}_{c}, \mathrm{MTT}_{A B}$, and $\mathrm{F}_{a b}$ form the third group.

As to GB-specified values (95\% probability value or the maximum), $s_{a b}, s_{b c}$, and $s_{c a}$ are corresponding the load power of the load processes $s_{\mathrm{L} a b}(t), s_{\mathrm{L} b c}(t)$ and, $s_{\mathrm{L} c a}(t)$ for feeders $\mathrm{F}_{a b}, \mathrm{~F}_{b c}$, and $\mathrm{F}_{c a}$, respectively. The minimum among them is denoted by

$s_{\min }=\min \left\{s_{a b}, s_{b c}, s_{c a}\right\}$.

The synthesis of three negative-sequence components is determined by differences between two larger load powers and the minimum load power. For instance, if the minimum load power $s_{\min }=s_{c a}$, the synthesis of negative-sequence components can be synthesized from $s_{a b}-s_{c a}$ and $s_{b c}-s_{c a}$ at $120^{\circ}$. In this case, the $\mathrm{ADA}_{b}$ in the group of feeder $\mathrm{F}_{c a}$ is not functioning, while the $\mathrm{ADA}_{a}$ in the group of feeder $\mathrm{F}_{b c}$ and the $\mathrm{ADA}_{c}$ in the group of feeder $\mathrm{F}_{a b}$ are functioning. Similar to the two line case, the calculated capacity of $\mathrm{ADA}_{a}$ can be obtained by substituting $s=s_{b c}-s_{c a}$ into Eq. (5) and that of $\mathrm{ADA}_{c}$ can be obtained by substituting $s=s_{a b}-s_{c a}$ into Eq. (5). The calculated capacity of the MTT should be added in $s_{\min }=s_{c a}$ after obtained from Eq. (5). Following a similar design process, all results can be obtained in the cases of $s_{\min }=s_{a b}$ and $s_{\min }=s_{b c}$. The final calculated capacities of $\mathrm{ADA}_{a}, \mathrm{ADA}_{b}$, and $\mathrm{ADA}_{c}$ should take the maximum among three cases of $s_{\min }=s_{c a}$, $s_{\min }=s_{a b}$, and $s_{\min }=s_{b c}$. It is notable that the closer the three lines' load power $s_{a b}, s_{b c}$, and $s_{c a}$ are, the smaller the NCD capacity required is. When $s_{a b}, s_{b c}$, and $s_{c a}$ are equal, the NCD capacity is zero, which means no negativesequence compensation is required.

When more than three lines are considered, the rule would be that the load to each of lines should be shared by three line voltages as equally as possible. The capacity of MTTs or the number of feeders can be increased, but the number of MTTs should be unchanged. Likewise, the number of backup MTTs should be kept unchanged.

\section{System's economy and reliability}

Merits in terms of reliability, economy, and technical performance of an AC traction power supply system (AC system, in short) proposed in this paper can be obtained by comparing with the existing DC traction power supply system (DC system, in short).

\subsection{Economy}

The DC system shares the same $35 \mathrm{kV}$ cable for the traction power supply, three-phase power, and single-phase lighting supply $[4,6]$ while the AC system uses the separate cables for them. But this should not add much extra cost. Compared with the DC system, the AC system can save the anti-stray current devices along the metro line, save rectifier units and DC switches at each substation, and save expensive energy storage devices or renewable energy feedback devices. Consequently the metro's underground equipment can be simplified, thus occupied area is reduced. In terms of integrated automatic control devices [4-6], both the DC and AC systems have the similar scale and costs. However, the AC system needs to have the NCDs, the cost for an AC-DC-C train is higher than that for a DC-AC train. Generally, one-time costs of an AC system may be close to that of a DC system, but the MSS installed capacity of an AC traction system is significantly lower than the capacity sum of all individual SSs. Thus power resources and costs of the basic electricity are saved. In an AC system, the renewable energy can be directly used in an efficient way, resulting in further saving of power cost. All of these merits to an AC system are beneficial to the economy and in line with the national policy of energy conservation and emission reduction.

\subsection{Reliability}

An AC system obviates rectifier units of a DC system and reduces the number of elements in series, so as to help 
improving reliability. The AC circuit breaker (switch), compared to the DC breaker, is less expensive and more reliable and durable. Moreover, the segmented power supply technology, the status identification, and the protection technology are well applicable to any complex power supply system and able to improve system's reliability significantly $[11,17]$. NCDs can have their reasonable backup. NCD's reliability is not less than that of the MTT in an MSS. Even in the case that all NCDs are out of service for a short period of time, the MTT can take advantages of its overload ability and continue to provide power supply. In short, the AC system has a higher reliability.

\subsection{Applicability}

The integrated automation system built upon the segmented power supply technology, status identification, and protection technology can allow more convenience for change of traction power supply mode, routine maintenance, or repair.

For an urban rail transit system powered by the AC system, because two lines or more than two lines can share one main transformer, each line only requires one MSS. Such a centralized power supply system can not only cut down the interface to the utility grid substantially but also facilitate daily management.

\section{Conclusions}

This paper has presented and discussed an AC traction power supply system that has the following salient features:

- An AC system avoids the stray current occurring in a DC system.

- Using the industrial frequency $\mathrm{AC}$, the given traction power supply system can connect to a utility grid directly and thus gains economic, reliable, and strong power supply.

- The use of cables meets the requirements of long distance, high capacity, without phase-splits for power supply.

- A train with an AC-DC-AC drive system and the AC traction motor is able to achieve better traction and more direct use of renewable energy.

Above features can solidify the application foundation of the proposed system in the urban rail transit, such as metro and light rail systems.

Several key technologies employed in the proposed system are discussed. The main work and the conclusion can be summarized as follows:
(1) The power supply structure, principle, equivalent circuit, and transmission ability of the CTN are described. The $35 \mathrm{kV}$ cable-OCS matching technique, and the OCS voltage grade selection are also discussed. Analysis shows that the CTN can meet not only the requirements of power supply capacity but also the distance demands for any metro or light rail lines.

(2) A segmentation technology for the traction network is discussed. Particular attentions are given to OCS segmentation, status identification, and protection methods. Using the segmentation technology for traction network, its operation and maintenance can be conveniently changed or scheduled. Faults can be detected and isolated timely so that their impacts can be limited to the minimum extent. As a result, the system's reliability has been improved greatly.

(3) In addition to the power supply scheme for one line railway, schemes for two or more lines railway where one MSS is shared are also given. For each of the schemes, the method for calculating the minimum capacity of the NCD is presented, which ultimately leads to an optimal power supply solution.

In summary, the proposed system, with good technical performance, good economy, and high reliability, provides a new promising alternative solution for urban rail transit, such as metro and light rail transit systems. Therefore, it is worthy of further research and investigation for the future.

Acknowledgement The author is grateful for numerous support from faculty members, colleagues, and students in School of Electrical Engineering, Southwest Jiaotong University, who participated or helped in this research work.

Open Access This article is distributed under the terms of the Creative Commons Attribution 4.0 International License (http:// creativecommons.org/licenses/by/4.0/), which permits unrestricted use, distribution, and reproduction in any medium, provided you give appropriate credit to the original author(s) and the source, provide a link to the Creative Commons license, and indicate if changes were made.

\section{References}

1. Cao JY (1956) The way of electrified railway in China, People's Daily, China (26 Nov, 1956) (in Chinese)

2. Cao JY (1983) Power supply system of electrified railway. Press of Chinese Railway, Beijing, pp 106-109 (in Chinese)

3. Mark Walter GK (1989) Power supply of electrified railway (trans: Yuan ZF, He QG). Press of Southwest Jiaotong University, Chengdu, pp 141-145 (in Chinese)

4. He WJ, Gao SB, Zhang SQ, Wang X (1998) Electric traction power supply and transforming technology. Press of Southwest Jiaotong University, pp 94-104 and 188-224 (in Chinese) 
5. Qian QQ (2000) Electrified railway computer monitoring technology. Press of Southwest Jiaotong University, Chengdu (in Chinese)

6. Yu SW, Yang XS, Han LX, Zhang W (2008) Urban rail transit power supply system design principles and applications. Press of Southwest Jiaotong University, Chengdu, pp 225-242 (in Chinese)

7. Lian JS (2001) Introduction to Electric Drive Locomotive. Press of Southwest Jiaotong University, Chengdu, pp 127-151 (in Chinese)

8. Li QZ, Zhang JS, He WJ (1988) Research on new power system for heavy haul traction. J China Railw Soc 4:23-31 (in Chinese)

9. Li QZ, Lian JS, Gao SB (2006) Electrified engineering of high speed railway. Press of Southwest Jiaotong University, Chengdu, pp 155-166 (in Chinese)

10. Li QZ (2006) Electrical analysis of traction substation and technology of comprehensive compensation. Press of Chinese Railway, Beijing, pp 72-93 (in Chinese)

11. Li QZ (2014) On new generation traction power supply system and its key technologies for electrified railways. J Southwest Jiaotong Univ 49(4):559-568 (in Chinese)
12. Tang XH (2006) Protection setting of loop power grid with voltage of $110 \mathrm{kV}$ and below. Rural Electr 27(9):28-30 (in Chinese)

13. Wang W, Gao K (2010) Analysis of loop problem of Beijing power grid. Water Resour Hydropower Northeast China 12:58-61 (in Chinese)

14. Encyclopedia of China's Power (Utility Grid Volume) (1995) China Electric Power Press, 1995, Beijing, pp 309-310 (in Chinese)

15. Qiou GY (1989) The circuit (2), 3rd edn. Higher Education Press, Beijing, pp 185-198 (in Chinese)

16. Li QZ, Yi D, He JM (2013) Analysis of traction power supply system with cables for electrified railways. J Southwest Jiaotong Univ 48(1):81-87 (in Chinese)

17. Li QZ, Chen MM, Huang YY (2014) Research of power supply state monitoring and control method of AT traction network for high-speed railway, Electric Railway, Supplement, pp. 11-15 (in Chinese)

18. Standardization Administration of the People's Republic of China (2008) GB/T15543-2008 power quality three-phase voltage unbalance (in Chinese) 